\title{
Self-perceived health in functionally independent older people: associated factors
}

Mónica Machón 1,2,3*, Itziar Vergara ${ }^{1,2,3}$, Miren Dorronsoro ${ }^{4,5,3}$, Kalliopi Vrotsou ${ }^{1,2,3}$ and Isabel Larrañaga 6,3

\begin{abstract}
Background: Self-perceived health (SPH) is a powerful indicator of the health status of elderly people. This issue has been widely studied in oldest populations considering altogether functionally independent and dependent individuals. The objective of this study was to describe SPH and to identify the main factors that have an impact on SPH in a sample of functionally independent community-dwelling older adults.

Methods: For this cross-sectional study, face-to-face interviews were carried out with non-institutionalized functionally independent older individuals in a northern region of Spain. Participants were asked: "Overall, you would say that your health is excellent, very good, good, fair or poor?". SPH responses were grouped in two categories: good and poor. Binary logistic regression was used to identify factors associated with poor SPH.

Results: A sample of 634 individuals was studied, of whom 55 \% were women. The mean age was 74.8 (SD 6.7) years. About $18 \%$ of the respondents rated their health as poor. In the multivariate model adjusted for age and sex, reported poor health was significantly associated with polypharmacy ( $\geq 3$ drugs per day) (OR: $5.76,95 \%$ Cl: 3.60-9.18), the presence of sensory impairment (OR: 1.87, $95 \%$ Cl: 1.15-3.04), bad sleep quality (OR:1.82, $95 \%$ Cl: 1.02-3.28), a bad nutrition pattern (OR: $2.37,95 \% \mathrm{Cl}: 1.08-5.21$ ), not engaging in cognitively stimulating activities (OR: 4.08, 95 \% Cl: 1.64-10.20), or group social activities (OR: 2.62, 95 \% Cl: 1.63-4.23).

Conclusions: The study indicates that several health and social variables are strongly related to SPH in independent community-dwelling older adults. This finding highlights the need for thorough assessment of factors related to SPH in older independent adults, this being essential to develop health-related programmes for promoting active and healthy ageing and to delay the onset of dependence in this population.
\end{abstract}

Keywords: Older adults, Self-perceived health, Ageing, Living conditions

\section{Background}

Population ageing is a worldwide trend in recent decades. In Spain, there were an estimated 7.9 million people (17 \% of the total population) over the age of 65 in 2014 and this figure is expected to reach 15.4 million ( $38 \%$ of the total population) by 2064 [1]. Active and healthy ageing (AHA) is a multi-factorial process that has become a health policy priority for local, national and international health authorities [2] in order to reduce the growing incidence of

\footnotetext{
* Correspondence: monica.machonsobrado@osakidetza.eus

'Unidad de Investigación de Atención Primaria-OSIs Gipuzkoa, Osakidetza,

San Sebastián, Spain

${ }^{2}$ Red de Investigación en Servicios de Salud en Enfermedades Crónicas

(REDISSEC), Madrid, Spain

Full list of author information is available at the end of the article
}

dependence. Self-perceived health (SPH) is included in one of the six group of determinants of the AHA model proposed by the World Health Organization (WHO) [3].

$\mathrm{SPH}$ is a complex measure that "represents a summary statement about the way in which numerous aspects of health, both subjective and objective, are combined within the perceptual framework of the individual respondent" [4]. It has been widely used, and is considered a valid and reliable indicator [5-7] of overall health status, a predictor of mortality and of health services use [8]. It is usually evaluated with a single item, asking individuals to rate their overall health on a scale from poor to excellent. Several 
alternative phrasings of the SPH question exist, but all have similar response patterns [9].

SPH in older adults has been found to be associated to sociodemographic characteristics (namely, sex, age, education, and income), chronic diseases and functional status [10-12]. Additional factors linked to SPH are social relationships [11-13] and neighborhood environment $[14,15]$. However, the effect magnitude of these factors varies depending on the study design, population and cultural context [16], and not all studies include all the above mentioned variables.

Functional status, in particular, is widely recognised to be a powerful determinant of SPH in older adults [10]. The assessment of functional status is a key element in healthcare. The detection of functionality loss can guide the implementation of health interventions aimed to avoid or delay the onset of dependence. Given the relevance of functional status as a factor of SPH, when it is studied at the same time with other related factors, the latter may become secondary with their role remaining undervalued when functional status is considered. Possibly, this is why less is known about how older people with good functional status perceive their health and the actual role of other factors related to SPH in this population. The assessment of factors beyond functional status may provide relevant information for the design and implementation of health promotion strategies.

This work is part of a larger study focused on understanding aspects of health and living conditions of community-dwelling elderly people, from a comprehensive perspective. The objectives of this study are to describe $\mathrm{SPH}$ in a functionally independent community-dwelling older population and to identify the main factors associated with $\mathrm{SPH}$ in this population group.

\section{Methods}

\section{Sample size}

A sample of 800 older individuals, from the province of Gipuzkoa (with a population of over 708,000 [17]) in Spain, was randomly selected using multistage sampling. The derived sample was representative of the Gipuzkoa elderly population in terms of age (65-74, 75-84 and $\geq 85$ years) and sex according to the Basque Health Survey data [18].

\section{Fieldwork process}

The data collection took place between January 2013 and February 2013. Trained interviewers conducted face-toface interviews with the selected individuals in their homes. The information was collected using a battery of 145 questions that explored health and living conditions (Table 1). It was constructed based on the published literature and the recommendations of a multidisciplinary expert panel. A partner, relative, friend or caregiver was allowed to be present during the interview if so desired by the participants to feel more confident. This did not interfere with the subjective questions (e.g., SPH), that should be answered only by the older person.

The study was approved by the Ethics Committee of Gipuzkoa Health Region. Written informed consent was obtained from all participants.

\section{Measurements}

SPH was the main outcome of interest. It was measured using a single item. "Overall, you would say that your health is...", with five response options: excellent; very good; good; fair; and poor. This item and response options have been widely used in previous research studies and health surveys $[13,14,16,18]$. All responses were grouped into two categories, good (excellent/very good/

Table 1 Dimensions assessed, main variables and scales administered to the participants in the full battery

\begin{tabular}{|c|c|}
\hline Health and living condition dimensions assessed & Main variables and scales used \\
\hline Sociodemographic characteristics & Age; sex; marital status; level of education; occupational status; income \\
\hline Health status & $\begin{array}{l}\text { Functional status (BI, and LS, respectively); self-perceived health; } \\
\text { health-related quality of life (EQ-5D); chronic diseases; drug prescriptions; } \\
\text { cognitive status (SPMSQ); depressive symptoms (GDS); falls; sensory impairment }\end{array}$ \\
\hline Lifestyle & Smoking status; drinking habits; sleep; diet; undernutrition (STARU); physical activity \\
\hline Social relationships & $\begin{array}{l}\text { Leisure activities, household composition; help and care given and received; } \\
\text { social capital (STS); social support from family and friends (LSNS); } \\
\text { functional social support (Duke-UNC FSSQ) }\end{array}$ \\
\hline Social services & Use of social services \\
\hline Health services & Use of health services \\
\hline Housing & $\begin{array}{l}\text { Housing tenure; facilities (lift, shower/bath, and mobile phone, among others); } \\
\text { housing condition; indoor and outdoor access }\end{array}$ \\
\hline Neighbourhood environment & Proximity of services; adequacy of community services \\
\hline
\end{tabular}

BI Barthel index, LS Lawton instrumental activities of daily living scale, EQ-5D EuroQol 5D questionnaire, SPMSQ short portable mental status questionnaire of Pfeiffer, GDS geriatric depression scale, STARU screening tool for assessing risk of undernutrition, STS social trust scale, LSNS Lubben social network scale, Duke-UNC FSSQ Duke-UNC functional social support questionnaire 
good) and poor (fair/poor); approach adopted by other researchers $[10,19]$.

The following individual, social and contextual variables were assessed.

First, in relation to the individual, sociodemographic characteristics were considered: sex, age, level of education, monthly family income and living arrangements.

Regarding health, several variables were studied. Cognitive status was measured with the Pfeiffer's Short Portable Mental Status Questionnaire [20, 21]. This test is composed of ten items and the total number of errors made in answering them is recorded. Functional status was assessed using the Barthel Index [22, 23] measuring ability to perform basic activities of daily living (BADL). This scale consists of ten items and its overall possible score ranges from 0 to 100 , with lower scores indicating more severe dependence. Diagnosed chronic conditions, including diabetes, hypertension, and cardiovascular disease, among others were recorded. Moreover, participants were asked about prescription drugs taken daily for the previous 2 weeks, considering polypharmacy if they took three or more [24]. Depressive symptoms were measured with the Geriatric Depression Scale $[25,26]$, which contains 15 items (10 positive and 5 negative). A score of 5 or above is considered to suggest the presence of depressive symptoms. Further, the number of falls occurred in the previous 12 months was recorded. Lastly, a variable was created indicating whether participants had difficulties with sensory-related abilities, considering hearing and vision, but also speech and chewing. The latter was considered part of the sensory-related abilities due to the fact that impaired masticatory abilities may have an impact on general health and quality of life [27]. Sensory impairment was defined as having difficulties with at least one of the aforementioned abilities.

Lifestyle habits analysed included participants' smoking status; whether they had done any physical activity in the previous 2 weeks; and the sleep quality, that is, whether they generally got a good night's sleep, good enough to feel well rested. Furthermore, a validated screening tool was used for assessing the risk of undernutrition [28, 29]. It is composed of nine items and its total score ranges from a minimum of 0 to a maximum of 26 points. A score of 7 or above was considered to indicate that a person was at risk of being undernourished or actually undernourished presenting a bad nutrition pattern.

Second, regarding social variables, we investigated how participants spent their leisure time, asking them how frequently in the last 12 months they had engaged in the following: cognitively stimulating activities (reading, listening to the radio); active leisure activities (going for a walk, taking care of a pet, gardening); social leisure activities (spending time with friends, attending sport events, going to a dance club, going to the cinema/theatre or a concert, going to a bar or out to have lunch/dinner); and group social activities (going on holiday). For the first three, individuals were considered as attending a certain type of activity if they participated in at least one of the activities of that group.

To assess social support, we used the Lubben Social Network Scale [30, 31]. This instrument includes six items about perceived social support from family and friends, with each response rated from 0 to 5 . The total score is an equally-weighted sum of scores on these six items. A higher score indicates a higher level of perceived social support. Results were dichotomized, using a cut-off point of less than 12 for the whole scale. Functional social support was measured using the 11-item Duke-UNC Functional Social Support Questionnaire [32, 33]. Responses are scored on a 5-point likert scale with higher scores indicating a higher level of social support [33]. A total score of $\leq 32$ was considered to indicate that the respondent had a low level of functional social support. Social capital was evaluated with a three-item scale used in the European Social Survey [34]. All questions were answered on an 11-point scale ranging from 0 representing "low trust" to 10 representing "high trust". In the constructed scale, total scores of up to 5 were considered to reflect low social trust and scores over 5 high social trust. Further, participants were asked to rate their social life, and responses were grouped into: satisfactory (very satisfactory/satisfactory), and unsatisfactory (unsatisfactory/very unsatisfactory).

Lastly, regarding context, participants were asked to state whether there were physical barriers or other obstacles to their mobility in their home (yes/no); and to rate the overall condition of their home, classifying this selfreported housing condition as good (good, very good or excellent) or poor (poor, fair). Community and neighbourhood resources were assessed using 21 items, based on the Checklist of Essential Features of Age-friendly Cities of the WHO [35], exploring the following features: outdoor spaces (pavements, green spaces, public toilets and streets), public buildings (elevators, toilets and ramps) and public transport (accessible vehicles, priority seats and drop-off spots). Answers were merged into two categories: none/very few/few vs. some/many/very many. Community services were considered adequate if the individual answered some/many/very many to questions about whether there were green spaces, public toilets, accessible public transport and priority seats on public transport. In addition, an item was included asking whether they walked or used transport to travel to a number of facilities.

Questions requiring a frequency response (e.g., number of prescription drugs consumed, cigarettes smoked last week) lacked close answers and were replied in a numerical form by the participants. No open reply opinion questions were asked in the study. 


\section{Exclusion criteria}

We excluded individuals with cognitive impairment, as this could compromise their ability to provide valid answers, and also those with dependence. Individuals were classified as a) having cognitive impairment, if they could read and made three or more errors, or if they were illiterate and made four or more errors, in Pfeiffer's Short Portable Mental Status Questionnaire [20, 21]; and b) dependent, if they obtained a Barthel Index score of $<95$ points [22, 23].

\section{Statistical analysis}

Categorical variables are presented as frequencies with percentages (\%) and continuous variables as means with standard deviations (SD). Chi-square or Fisher's exact tests were implemented for comparing categorical variables and Student's $t$-test was used in two-group comparisons of continuous variables. Univariate and multivariate binary logistic regression models adjusted for age and sex were fitted for the SPH outcome. All variables with $p$ values $<0.10$ at the univariate stage were considered during the multivariate analysis phase. Both backward and forward regression analysis was performed. The model results are presented as odds ratios (OR) with their respective $95 \%$ confidence intervals (95\% CI) and $p$-values. Differences were considered statistically significant if $p$-values were $<0.05$. The correlation matrix of estimated parameters, their eigenvalues and proportion of variation were examined for assessing collinearity presence [36]. The Hosmer-Lemeshow test and R-square and area under the curve (AUC) values are given for the final model. All analyses were performed with SAS 9.3 software.

\section{Results}

Of the 800 individuals selected, 125 were excluded from the analysis for cognitive impairment and 41 for BADL dependence. Those excluded were mostly women (67\%), were older $(p<0.0001)$ and had poorer health, with a higher percentage having $\geq 3$ chronic conditions $(p<$ $0.0001)$ and taking $\geq 3$ prescription drugs $(p<0.0001)$ compared to the included individuals. Thus, the final sample included in this study consisted of 634 independent community-dwelling older adults. The mean age of the individuals included was 74.8 (SD 6.7) years and more than half $(55 \%)$ of them were women. A total of 126 subjects were accompanied during the interview and 12 (2\%) of the participants answered the battery of questions with some help by the accompanying person. The five SPH categories were replied as follows: excellent $(n=38$ participants), very good $(n=147)$, good $(n=333)$, fair $(n=105)$ and poor $(n=11)$. Thus, about $18 \%$ of the respondents rated their $\mathrm{SPH}$ as poor.
From an individual perspective, those with a higher level of education were more likely to report a good SPH $(p=$ $0.035)$, as were those with higher monthly incomes $(p=$ 0.053 ) (Table 2). In addition, those presenting $\geq 3$ chronic diseases and taking $\geq 3$ drugs daily $(p<0.0001)$, having depressive symptoms $(p<0.0001)$, sensory impairment $(p=$ $0.0001)$, bad nutrition pattern $(p=0.0001)$ and failing to get a good night's sleep $(p=0.002)$ were more likely to report a poor SPH.

From a social point of view, the percentage of those reporting good health was significantly higher among those who engaged in cognitively stimulating activities $(p<$ $0.0001)$, social leisure activities $(p=0.001)$ or group social activities $(p<0.0001)$, with high social trust $(p=0.017)$ and satisfactory self-perceived social life $(p<0.0001)$ than it was for those who did not engage in the social activities considered, had low social trust or unsatisfactory social life.

Regarding contextual variables, individuals without obstacles inside their home $(p=0.031)$ and with a supermarket within walking distance $(p=0.003)$ were more likely to perceive their health as good.

No statistically significant differences were observed by age or sex, living arrangements, history of falls, social support, physical activity, smoking status, housing condition, active leisure activities or adequacy of community services (Table 2).

At the multivariate analysis stage, polypharmacy, sensory impairment, bad sleep quality, bad nutrition pattern and not engaging in certain types of leisure activities were identified as the set of factors that best explained SPH. Specifically, according to this model (Table 3), respondents with polypharmacy were more likely to report poor health (OR: 5.76, 95 \% CI: 3.60-9.18). Further, respondents with at least one sensory impairment, those with bad sleep quality and those with bad nutrition pattern were around twice likely to rate their $\mathrm{SPH}$ as poor (OR: 1.87, $95 \% \mathrm{CI}: 1.15-3.04$; OR: 1.82 , 95 \% CI: 1.02-3.28; and OR: 2.37, 95 \% CI: $1.08-$ 5.21 , respectively). Finally, individuals who did not engage in cognitively stimulating activities or group social activities were also more likely to rate their SPH as poor (OR: 4.08, 95 \% CI: $1.64-10.20$ and OR: 2.62, 95 \% CI: 1.63-4.23, respectively). Collinearity diagnostics did not reveal any problems with the included variables. The AUC of this model was 0.810 , suggesting good discriminatory capacity [37].

\section{Discussion}

This study aimed to assess the role of various individual, social and contextual factors in the assessment of SPH in independent older people without cognitive impairment. For this purpose, individuals were considered independent if they had good cognitive status and BADL function. In past research [11], people with cognitive impairment have also been excluded, given their level of deterioration could 
Table 2 Characteristics of older people according to their self-perceived health

\begin{tabular}{|c|c|c|c|}
\hline \multirow[b]{2}{*}{ Variables } & \multicolumn{3}{|c|}{ Self-perceived health } \\
\hline & Poor $(n=116)$ & Good $(n=518)$ & $p$-value \\
\hline \multicolumn{4}{|l|}{ Sex } \\
\hline Men & $47(16)$ & $238(84)$ & \multirow[t]{2}{*}{0.288} \\
\hline Women & $69(20)$ & $280(80)$ & \\
\hline Age (years), mean (SD) & $74.9(6.3)$ & $74.8(6.8)$ & 0.865 \\
\hline \multicolumn{4}{|l|}{ Level of education } \\
\hline Primary or lower & $103(20)$ & $416(80)$ & \multirow[t]{2}{*}{0.035} \\
\hline Secondary or higher & $13(11)$ & $101(89)$ & \\
\hline \multicolumn{4}{|l|}{ Monthly family income $(€)$} \\
\hline$\leq 1500$ & $68(20)$ & $277(80)$ & \multirow[t]{2}{*}{0.053} \\
\hline$\geq 1501$ & $18(12)$ & $127(88)$ & \\
\hline \multicolumn{4}{|l|}{ Living arrangements } \\
\hline Alone & $29(18)$ & $130(82)$ & \multirow[t]{2}{*}{0.974} \\
\hline With others & $87(18)$ & $387(82)$ & \\
\hline \multicolumn{4}{|c|}{ Number of diagnosed chronic diseases } \\
\hline $0-2$ & $45(10)$ & $403(90)$ & \multirow[t]{2}{*}{$<0.0001$} \\
\hline$\geq 3$ & $71(38)$ & $115(62)$ & \\
\hline \multicolumn{4}{|l|}{ Number of drugs taken daily } \\
\hline $0-2$ & $38(9)$ & $384(91)$ & \multirow[t]{2}{*}{$<0.0001$} \\
\hline$\geq 3$ & $78(37)$ & $134(63)$ & \\
\hline \multicolumn{4}{|l|}{ GDS score } \\
\hline Not depressive symptoms $(<5)$ & $86(15)$ & $470(85)$ & \multirow[t]{2}{*}{$<0.0001$} \\
\hline Depressive symptoms ( $\geq 5$ ) & $30(38)$ & $48(62)$ & \\
\hline \multicolumn{4}{|l|}{ Falls in the previous 12 months } \\
\hline 0 & $91(18)$ & $424(82)$ & \multirow[t]{2}{*}{0.396} \\
\hline$\geq 1$ & $25(21)$ & $94(79)$ & \\
\hline \multicolumn{4}{|l|}{ Sensory impairment } \\
\hline$\geq 1$ & $46(28)$ & $117(72)$ & \multirow[t]{2}{*}{0.0001} \\
\hline 0 & $70(15)$ & $401(85)$ & \\
\hline \multicolumn{4}{|l|}{ Smoker } \\
\hline Yes & $7(17)$ & $35(83)$ & \multirow[t]{2}{*}{0.774} \\
\hline No & $109(18)$ & $482(82)$ & \\
\hline \multicolumn{4}{|c|}{ Physical activity in the previous 2 weeks } \\
\hline Yes & $94(18)$ & $437(82)$ & \multirow[t]{2}{*}{0.380} \\
\hline No & $22(21)$ & $81(79)$ & \\
\hline \multicolumn{4}{|l|}{ Sleep quality } \\
\hline Good & $89(16)$ & $463(84)$ & 0.002 \\
\hline Bad & $27(33)$ & $55(67)$ & \\
\hline Nutrition pattern (STARU) & & & \\
\hline Good $(<7)$ & $101(17)$ & $498(83)$ & 0.0001 \\
\hline $\operatorname{Bad}(\geq 7)$ & $15(43)$ & $20(57)$ & \\
\hline Cognitively stimulating activities & & & \\
\hline Yes & $104(17)$ & $504(83)$ & $<0.0001$ \\
\hline No & $12(46)$ & $14(54)$ & \\
\hline
\end{tabular}


Table 2 Characteristics of older people according to their self-perceived health (Continued)

\begin{tabular}{|c|c|c|c|}
\hline \multicolumn{4}{|l|}{ Active leisure activities } \\
\hline Yes & $110(18)$ & $503(82)$ & \multirow[t]{2}{*}{0.245} \\
\hline No & $6(29)$ & $15(71)$ & \\
\hline \multicolumn{4}{|l|}{ Social leisure activities } \\
\hline Yes & $90(16)$ & $462(84)$ & \multirow[t]{2}{*}{0.001} \\
\hline No & $26(32)$ & $56(68)$ & \\
\hline \multicolumn{4}{|l|}{ Group social activities } \\
\hline Yes & $41(12)$ & $305(88)$ & \multirow[t]{2}{*}{$<0.0001$} \\
\hline No & $75(26)$ & $211(74)$ & \\
\hline \multicolumn{4}{|l|}{ LSNS score } \\
\hline Low level of perceived social support $(<12)$ & $27(19)$ & $113(81)$ & \multirow[t]{2}{*}{0.732} \\
\hline High level of perceived social support $(\geq 12)$ & $89(18)$ & $405(82)$ & \\
\hline \multicolumn{4}{|l|}{ Duke-UNC FSSQ score } \\
\hline Low level of functional social support $(\leq 32)$ & $11(15)$ & $61(85)$ & \multirow[t]{2}{*}{0.416} \\
\hline High level of functional social support (>32) & $105(19)$ & $457(81)$ & \\
\hline \multicolumn{4}{|l|}{ STS } \\
\hline Low social trust $(\leq 5)$ & $49(24)$ & $159(76)$ & \multirow[t]{2}{*}{0.017} \\
\hline High social trust (>5) & $67(16)$ & $359(84)$ & \\
\hline \multicolumn{4}{|l|}{ Self-perceived social life } \\
\hline Unsatisfactory & $18(42)$ & $25(58)$ & \multirow[t]{2}{*}{$<0.0001$} \\
\hline Satisfactory & $98(17)$ & $493(83)$ & \\
\hline \multicolumn{4}{|l|}{ Obstacles inside the home } \\
\hline Yes & $15(29)$ & $36(71)$ & \multirow[t]{2}{*}{0.031} \\
\hline No & $100(17)$ & $480(83)$ & \\
\hline \multicolumn{4}{|l|}{ Self perceived housing condition } \\
\hline Good & $101(17)$ & $477(83)$ & \multirow[t]{2}{*}{0.085} \\
\hline Poor & $15(27)$ & $41(73)$ & \\
\hline \multicolumn{4}{|l|}{ Adequate community services } \\
\hline Yes & $15(14)$ & $95(86)$ & \multirow[t]{2}{*}{0.164} \\
\hline No & $101(19)$ & $423(81)$ & \\
\hline \multicolumn{4}{|l|}{ Supermarket within walking distance } \\
\hline Yes & $83(16)$ & $432(84)$ & \multirow[t]{2}{*}{0.003} \\
\hline No & $33(28)$ & $86(72)$ & \\
\hline
\end{tabular}

Numbers are $\mathrm{n}(\%)$ unless otherwise stated. Row percentages are presented. When missing data exist, frequencies do not add up to column totals. GDS geriatric depression scale, STARU screening tool for assessing risk of undernutrition, LSNS Lubben social network scale, Duke-UNC FSSQ Duke-UNC functional social support questionnaire, STS social trust scale

interfere with their ability to provide valid answers. Inability to perform daily life activities was also an exclusion criteria applied in a previous older people study [11]. Therefore, the approach followed in the present study can provide further research information on which other factors besides autonomy are associated with SPH.

The multivariate model that best explained poor SPH in our data, after adjusting for age and sex, included the following variables: polypharmacy, having sensory impairment, bad sleep quality, bad nutrition pattern and not engaging in cognitively stimulating activities or group social activities. These results are consistent with the complex definition of AHA [3, 38, 39]. The presented model includes factors related to individual health status, like polypharmacy, clearly related to the presence of chronic diseases. Also three elements rarely relevant in younger populations, namely, sensory problems, sleep quality and nutrition patterns [38-40]. Additionally, our model includes two variables related to enjoyable activities: cognitively stimulating activities and group social activities. As 
Table 3 Factors associated with poor self-perceived health in older people. Multivariate logistic regression model results

\begin{tabular}{|c|c|c|}
\hline Variables & OR $(95 \% \mathrm{Cl})$ & $p$-value \\
\hline Age & $0.96(0.93-0.99)$ & 0.037 \\
\hline \multicolumn{3}{|l|}{ Sex } \\
\hline Women & 1.00 & \multirow[t]{2}{*}{0.979} \\
\hline Men & $0.99(0.62-1.58)$ & \\
\hline \multicolumn{2}{|c|}{ Number of drugs taken daily } & \multirow[t]{3}{*}{$<0.0001$} \\
\hline $0-2$ & 1.00 & \\
\hline$\geq 3$ & $5.76(3.60-9.18)$ & \\
\hline \multicolumn{3}{|c|}{ Sensory impairment } \\
\hline 0 & 1.00 & \multirow[t]{2}{*}{0.019} \\
\hline$\geq 1$ & $1.87(1.15-3.04)$ & \\
\hline \multicolumn{3}{|c|}{ Sleep quality } \\
\hline Good & 1.00 & \multirow[t]{2}{*}{0.045} \\
\hline Bad & $1.82(1.02-3.28)$ & \\
\hline \multicolumn{3}{|c|}{ Nutrition pattern (STARU) } \\
\hline Good & 1.00 & \multirow[t]{2}{*}{0.032} \\
\hline Bad & $2.37(1.08-5.21)$ & \\
\hline \multicolumn{3}{|c|}{ Cognitively stimulating activities } \\
\hline Yes & 1.00 & \multirow[t]{2}{*}{0.003} \\
\hline No & $4.08(1.64-10.20)$ & \\
\hline \multicolumn{3}{|c|}{ Group social activities } \\
\hline Yes & 1.00 & \multirow[t]{2}{*}{$<0.0001$} \\
\hline No & $2.62(1.63-4.23)$ & \\
\hline
\end{tabular}

STARU screening tool for assessing risk of undernutrition, OR odds ratio, $95 \%$ IC $95 \%$ confidence interval; OR $>1$ indicate higher odds of poor $\mathrm{SPH} ; \mathrm{OR}<1$ indicate lower odds of poor SPH; Estimates are based on $n=632$ participants due to missing values; Model diagnostics AUC $=0.810$; Hosmer-Lemeshow- $p$ value $=0.152 ; R$-square $=0.169 ;$ Max-Rescaled 0.318

far as cognitively stimulating activities are concerned, reading may be associated with educational attainment, but not listening to the radio. This finding may indicate the importance of maintaining mental vitality into late adulthood as a component of healthy ageing. A WHO report of active ageing [39] encourages older people to keep learning to maintain cognitive capacities. Other relevant aspects associated with a satisfactory cognitive ageing are the absence of stress in older people, found to be related to a lower deleterious impact in the brain [41], and brain reserve, where higher mental activities have been found to be associated with less cognitive decline [42]. Group social activities are a multicomponent variable that reflects a good level of interest in socialising and maintaining a social network. Both cognitively stimulating activities and group social activities are factors that may contribute to improving quality of life of older people, being the latter also important to enhancing social connectedness [43]. Previous works have also examined the relation between social activities, social participation or social integration and SPH $[11,13]$. Although different social activities have been studied, such as, participation in recreational activities at a public hall or community association for the aged [11], in a hobby association [11], in educational and cultural activities [13], in sports or physical activities with other people, in family/friendship activities outside the household [13], or going to a social centre for the elderly [12]; in all cases, participants had better SPH than nonparticipants.

Age, sex, education, income, chronic diseases, functional status and mental health have also been found to be linked to SPH in previous studies [10-12]. In our data of functionally independent subjects, all other factors but sex were associated with SPH in the univariate analysis stage. The lack of association with sex is completely unexpected and is probably due to the selection criteria applied, in particular, the exclusion of dependent individuals, with dependence being more common among women. However, sex was not a predictor of SPH in a previous study of older Thai people [10].

On the other hand, our finding that good night sleep is related to a better SPH is consistent with a cohort study of non-institutionalized Spanish adults aged 60 years or more where extreme sleep durations ( $\leq 5$ or $\geq 10 \mathrm{~h}$ ) were associated with a poorer health-related quality of life [44]. Sleep is a vital physiological process and the mechanisms implicated in its duration and quality are complex and multidimensional [45]. Several changes are seen in older people, as aged individuals, are more prone to wake up at earlier times or experienced an involuntary reduction of their total sleep duration [46]. Finally, disturbances in the circadian rhythm have been associated with a worse health [47]. All the above reveals the importance of maintaining adequate sleep patterns in this population group.

Sensory-related abilities are also found to be related to $\mathrm{SPH}$. This is in concordance with a study of nondisabled older people living alone [11], where problems in visual, hearing and chewing abilities were associated with poor SPH. One of the challenges of an ageing population according to $\mathrm{WHO}$ are precisely vision and hearing losses as well as oral health [39]. The WHO urges countries to develop policies and programmes to prevent visual and hearing impairment and promote good oral health habits [39]. Similarly, the development of guidelines promoting an adequate nutrition is a need to solve eating problems, understanding as such both under nutrition and excess energy intakes [39].

Another difference with other published studies [14, 15] is the lack of associations with neighbourhood characteristics (adequate community services and proximity to a number of facilities), either in the univariate (except for proximity to supermarket) or in the multivariate analysis. One possible explanation is that people with poor SPH may have a different perception of their neighbourhood 
environment than those in good health [48] and may not even perceive these problems as they go out less frequently. It is also likely that, if individuals live in the same neighbourhood for a long time, they get used to the environment. Another plausible justification may be that the measure created to analyse this factor was not suitable for use in older people. Finally, it is also important to consider that the questions used to assess community services were based on WHO age friendly city criteria [35] and were not part of an specific or validated questionnaire. Therefore, further research will be conducted exploring this issue and, perhaps, new tools should be developed to explore elderly peoples' perception of their communities.

Several strengths of our study deserve to be highlighted. Firstly, the study was based on a large sample of community-dwelling older people and we have investigated the association between SPH and multiple dimensions of health and living conditions, using a wide range of individual, social and contextual variables. Another important characteristic of this study was the collaborative participation of the multidisciplinary expert panel. They reviewed and commented on the draft of the questionnaire and helped with the development of the final version, from a comprehensive multifactorial perspective.

On the other hand, there are limitations that should be recognised. The study design was cross-sectional, and hence, it is not possible to establish cause-effect relationships. Nonetheless, as stated by Giron et al. [19], this type of research is necessary to help us understand factors that determine or alter health. Moreover, the battery of questions developed is extensive (145 items that were responded in approximately $60 \mathrm{~min}$ ) and may explain why individuals who agreed to participate tended to have a relatively good functional status. Another limitation of this study is that the data were collected via face-to-face interviews by 20 interviewers. So, although interviewers were trained, there is always a possibility that the data collection technique varied, as was explained in the study of Tajvar et al. [49]. Nevertheless, face-to-face interviews are the recommended way to collect information in the case of long questionnaires, and generally obtain higher response rates than postal or telephone interviews [50]. Even though information of chronic conditions was self-reported by the individuals and thus may affect the accuracy of information, it is an approach used in similar previous studies $[10,11]$. It is also important to discuss about the representativeness of the sample. The original sample (800 individuals) was representative of the Gipuzkoa elderly population according to sex and age. The exclusion of non-independent and cognitively impaired individuals did not cause major alterations to the percentage of the considered variables, as in the finally included sample (634 individuals) all categories were between 1 and $5 \%$ of the original sample. Finally, missing data was not a particular problem in the current study but for the income level variable, which $22 \%$ of the sample did not reply. It is likely that full information on this very variable would have allowed for further explorations.

\section{Conclusions}

In conclusion, several different factors related to health and social variables account for the $\mathrm{SPH}$ of older people besides autonomy. Among them, the study found that drugs taken per day, sensory-related abilities, sleep quality, nutrition and leisure activities were the most strongly associated with SPH in a functionally independent elder population. In this sense, our results confirm the multidimensional nature of $\mathrm{SPH}$ and are consistent with those of other studies. Our work highlights the need for a thorough assessment of factors associated with SPH in older adults, this being essential for developing health-related programmes that promote active and healthy ageing.

\section{Abbreviations \\ AHA: active and healthy ageing; BADL: basic activities of daily living: BI: Barthel index; Cl: confidence intervals; Duke-UNC FSSQ: Duke-UNC functional social support questionnaire; EQ-5D: EuroQol 5D questionnaire; GDS: geriatric depression scale; LS: Lawton instrumental activities of daily living scale; LSNS: Lubben social network scale; OR: odds ratios; SD: standard deviation; SPH: self-perceived health; SPMSQ: short portable mental status questionnaire of Pfeiffer; STARU: screening tool for assessing risk of undernutrition; STS: social trust scale; WHO: World Health Organization.}

\section{Competing interests}

The authors declare that they have no competing interests.

\section{Authors' contributions}

$M M, I V$, IL and KV participated in the design of the study. KV performed the statistical analysis. MM, KV, IV, MD and IL drafted the manuscript. All authors participated in the interpretation of the data and read and approved the final manuscript.

\section{Acknowledgments}

We thank the participants in the study for their time and effort. The authors also want to thank the expert panel for recommendations that were instrumental in the development of the questionnaire: Juan Aitor Thalamas, Gemma Estévez, Merche González, Maite de Diego, María Victoria García, Unai Martín and Alejandro Morlán. Finally, we would like to express our gratitude to Bakarne Aguirre, for her help in the management of the project.

\section{Funding}

This work was supported by the Basque Foundation for Social and Health Care Innovation-ETORBIZI (Grant- BIOEFF11/FVSS/3.1).

\section{Author details}

'Unidad de Investigación de Atención Primaria-OSIs Gipuzkoa, Osakidetza, San Sebastián, Spain. ${ }^{2}$ Red de Investigación en Servicios de Salud en Enfermedades Crónicas (REDISSEC), Madrid, Spain. ${ }^{3}$ Instituto de Investigación Sanitaria Biodonostia, San Sebastián, Spain. ${ }^{4}$ Dirección de Salud Pública y Adicciones, Gobierno Vasco, Vitoria, Spain. ${ }^{5}$ CIBER Epidemiología y Salud Pública (CIBERESP), Madrid, Spain. ${ }^{6}$ Departamento de Salud, Delegación Territorial de Gipuzkoa, Gobierno Vasco, San Sebastián, Spain.

Received: 8 November 2015 Accepted: 3 March 2016

Published online: 09 March 2016

References

1. Spanish National Statistics Institute. Demography and population: population figures and demographic censuses. 2015.http://www.ine.es/en/ inebmenu/mnu_cifraspob_en.htm. Accessed18 Aug 2015. 
2. Bousquet J, Michel J, Strandberg T, Crooks G, lakovidis I, Iglesia M. The European Innovation Partnership on Active and Healthy Ageing: the European Geriatric Medicine introduces the EIP on AHA Column. Eur Geriatr Med. 2014;5:361-2

3. Paul C, Ribeiro O, Teixeira L. Active Ageing: An Empirical Approach to the WHO Model. Curr Gerontol Geriatr Res. 2012;2012:382972.

4. Tissue T. Another look at self-rated health among the elderly. J Gerontol. 1972;27:91-4.

5. Martikainen P, Aromaa A, Heliovaara M, Klaukka T, Knekt P, Maatela J, et al. Reliability of perceived health by sex and age. Soc Sci Med. 1999:48:1117-22.

6. Lundberg O, Manderbacka K. Assessing reliability of a measure of self-rated health. Scand J Soc Med. 1996;24:218-24.

7. Cousins SO. Validity and reliability of self-reported health of persons aged 70 and older. Health Care Women Int. 1997;18:165-74.

8. Jylha M. What is self-rated health and why does it predict mortality? Towards a unified conceptual model. Soc Sci Med. 2009;69:307-16.

9. Fayers PM, Sprangers MA. Understanding self-rated health. Lancet. 2002;359:187-8.

10. Haseen F, Adhikari R, Soonthorndhada K. Self-assessed health among Thai elderly. BMC Geriatr. 2010;10:30.

11. Sun W, Watanabe M, Tanimoto $Y$, Shibutani $T$, Kono R, Saito M, et al. Factors associated with good self-rated health of non-disabled elderly living alone in Japan: a cross-sectional study. BMC Public Health. 2007;7:297.

12. Zunzunegui MV, Kone A, Johri M, Beland F, Wolfson C, Bergman H. Social networks and self-rated health in two French-speaking Canadian community dwelling populations over 65. Soc Sci Med. 2004;58:2069-81.

13. Gilmour H. Social participation and the health and well-being of Canadian seniors. Health Rep. 2012;23:23-32.

14. Bowling A, Barber J, Morris R, Ebrahim S. Do perceptions of neighbourhood environment influence health? Baseline findings from a British survey of aging. J Epidemiol Community Health. 2006;60:476-83.

15. Roh S, Jang Y, Chiriboga DA, Kwag KH, Cho S, Bernstein K. Perceived neighborhood environment affecting physical and mental health: a study with Korean American older adults in New York City. J Immigr Minor Health. 2011;13:1005-12.

16. Pfarr C, Schmid A, Schneider U. Reporting heterogeneity in self-assessed health among elderly Europeans. Health Econ Rev. 2012;2:21.

17. Basque Statistic Institute (EUSTAT). Population of the Basque Country by province, according to large age groups and sex. 2014.http://en.eustat.es/ ci_ci/estadisticas/tema_159/opt_0/tipo_1/temas.html\#axzz3QlaB2Htb. Accessed 18 Aug 2015.

18. Departamento de Salud. Encuesta de Salud del País Vasco. 2013.http:// www.osakidetza.euskadi.eus/r85-pkpubl01/es/contenidos/informacion/ encuesta_salud/es escav/encuesta_salud.html. Accessed 18 Aug 2015.

19. Giron P. Is age associated with self-rated health among older people in Spain? Cent Eur J Public Health. 2012;20:185-90.

20. Pfeiffer E. A short portable mental status questionnaire for the assessment of organic brain deficit in elderly patients. J Am Geriatr Soc. 1975;23:433-41.

21. Martínez de la Iglesia J, Dueñas Herrero R, Onís Vilches MC, Aguado Taberné C, Albert Colomer C, Luque Luque R. Adaptación y validación al castellano del cuestionario de Pfeiffer (SPMSQ) para detectar la existencia de deterioro cognitivo en personas mayores de 65 años. Med Clin (Barc). 2001;117:129-34.

22. Mahoney Fl, Barthel DW. Funciontal evaluation: the Barthel index. Md State Med J. 1965;14:61-5

23. Baztán JJ, Pérez del Molino J, Alarcón T, San Cristóbal E, Izquierdo G, Manzarbeitia J. Indice de Barthel: Instrumento válido para la valoración funcional de pacientes con enfermeadad cerebrovascular. Rev Esp Geriatr Gerontol. 1993;28:32-40.

24. Fulton MM, Allen ER. Polypharmacy in the elderly: a literature review. J Am Acad Nurse Pract. 2005;17:123-32.

25. Sheikh J, Yesavage J. Geriatric Depression Scale (GDS). Recent evidence and development of a shorter version. Clin Gerontol. 1986;5:165-72.

26. Martínez de la Iglesia J, Onís Vilches MC, Dueñas HR, Albert CC, Aguado TC, Luque LR. Versión española del cuestionario de Yesavage abreviado (GDS) para el despistaje de depresión en mayores de 65 años: adaptación y validación. Medifam. 2002;12:620-30.

27. Kimura $Y$, Wada T, Ishine M, Ishimoto $Y$, Kasahara $Y$, Hirosaki M, et al. Communitydwelling elderly with chewing difficulties are more disabled, depressed and have lower quality of life scores. Geriatr Gerontol Int. 2009;9:102-4.
28. Ward J, Close J, Little J, Boorman J, Perkins A, Coles SJ, et al. Development of a screening tool for assessing risk ofundernutrition in patients in the community. J Hum Nutr Dietet. 1998;11:323-30.

29. Morillas J, García-Talavera N, Martín-Pozuelo G, Reina AB, Zafrilla P. Detección del riesgo de desnutrición en ancianos no institucionalizados. Nutr Hosp. 2006;21:650-6.

30. Lubben J, Blozik E, Gillmann G, lliffe S, von Renteln KW, Beck JC, et al. Performance of an abbreviated version of the Lubben Social Network Scale among three European community-dwelling older adult populations. Gerontologist. 2006;46:503-13.

31. Spanish Lubben Social Network Scale. The Trustees of Boston College 2013. http://www.bc.edu/schools/gssw/lubben/translations/_jcr_content/content/ download/file.res/Spanish\%20LSNSR.pdf. Accessed 29 Oct 2015.

32. Broadhead WE, Gehlbach SH, de Gruy FV, Kaplan BH. The Duke-UNC Functional Social Support Questionnaire. Measurement of social support in family medicine patients. Med Care. 1988;26:709-23.

33. Bellón Saameño JA, Delgado SA, Luna del Castillo JD, Lardelli Claret P. Validez y fiabilidad del cuestionario de apoyo social functional Duke-UNC 11. Aten Primaria. 1996;18:153-63.

34. Poortinga W. Social capital: an individual or collective resource for health? Soc Sci Med. 2006;62:292-302.

35. World Health Organization. Checklist of Essential Features of Age-friendly Cities. 2014.http://www.who.int/ageing/publications/Age_friendly_cities_ checklist.pdf?ua=1 .Accessed 18 Aug 2015.

36. Field A. Discovering statistics using IBM SPSS Statistics (third edition). London: Sage publications; 2009.

37. Steyerberg EW, Vickers AJ, Cook NR, Gerds T, Gonen M, Obuchowski N, et al Assessing the performance of prediction models: a framework for traditional and novel measures. Epidemiology. 2010;21:128-38.

38. Bousquet J, Kuh D, Bewick M, Strandberg T, Farrell J, Pengelly R, et al. Operative definition of active and healthy ageing (AHA): Meeting report. Montpellier October 20-21, 2014. Eur Geriatr Med. 2015;6:196-200.

39. Report of the World Health Organization. Active ageing: a policy framework. Aging Male. 2002;5:1-37

40. Depp CA, Jeste DV. Definitions and predictors of successful aging: a comprehensive review of larger quantitative studies. Am J Geriatr Psychiatr. 2006;14:6-20.

41. Depp CA, Harmell A, Vahia IV. Successful cognitive aging. Curr Top Behav Neurosci. 2012;10:35-50.

42. Valenzuela MJ, Sachdev P. Brain reserve and cognitive decline: a nonparametric systematic review. Psychol Med. 2006;36:1065-73.

43. Toepoel V. Ageing, Leisure, and Social Connectedness: How could Leisure Help Reduce Social Isolation of Older People? Soc Indic Res. 2013;113:355-72.

44. Faubel R, Lopez-Garcia E, Guallar-Castillon P, Balboa-Castillo T, GutierrezFisac JL, Banegas JR, et al. Sleep duration and health-related quality of life among older adults: a population-based cohort in Spain. Sleep. 2009;32:1059-68.

45. Bixler E. Sleep and society: an epidemiological perspective. Sleep Med. 2009; 10 Suppl 1:S3-6.

46. Crowley K. Sleep and sleep disorders in older adults. Neuropsychol Rev. 2011:21:41-53.

47. Popa-Wagner A, Catalin B, Buga AM. Novel putative mechanisms to link circadian clocks to healthy aging. J Neural Transm (Vienna). 2015;122 Suppl 1:S75-82.

48. Parra DC, Gomez LF, Sarmiento OL, Buchner D, Brownson R, Schimd T, et al Perceived and objective neighborhood environment attributes and health related quality of life among the elderly in Bogota, Colombia. Soc Sci Med. 2010;70:1070-6

49. Tajvar M, Arab M, Montazeri A. Determinants of health-related quality of life in elderly in Tehran, Iran. BMC Public Health. 2008;8:323.

50. McColl E, Jacoby A, Thomas L, Soutter J, Bamford C, Steen N, et al. Design and use of questionnaires: a review of best practice applicable to surveys of health service staff and patients. Health Technol Assess. 2001;5:1-256. 